\title{
Influence of waterpipe smoking on cardiac autonomic function at rest and following high-intensity anaerobic exercise
}

\author{
Mehdi Ahmadian",*, Saeed Ghorbani' ${ }^{1}$, Valiollah Dabidi Roshan ${ }^{2}$, and Anthony S. Leicht ${ }^{3}$ \\ ${ }^{I}$ Department of Physical Education and Sport Sciences, Aliabad Katoul Branch, Islamic Azad University, Aliabad Katoul, \\ Iran; ${ }^{2}$ Department of Sport Physiology, University of Mazandaran, Babolsar, Iran; and ${ }^{3}$ Sport and Exercise Science, James \\ Cook University, Townsville, Australia
}

Copyright: (c) 2018 M. Ahmadian et al. This is an open access article licensed under the Creative Commons Attribution License (http://creativecommons.org/licenses/by/4.0/).

Background: Waterpipe (WP) smoking has been linked to reduced birth weight, genetic damage, and respiratory and cardiovascular disease, possibly via enhanced exposure to carbon monoxide and volatile aldehydes. Objective: The effect of long-term WP smoking on cardiac autonomic function at rest and following exercise was investigated. Methods: Twenty males volunteered and were divided into two groups: WP smokers (WPS) and non-smokers (NSmk). Heart rate variability (HRV) at rest and following (10 mins) Wingate test was examined. Additionally, heart rate recovery (HRR) at 1 (HRR1), 2 (HRR2) and 5 mins (HRR5) post-Wingate test were considered. Results: At rest, the WPS group exhibited a significantly greater heart rate and lower standard deviation of RR intervals. The WPS group experienced a significantly greater peak heart rate while the post-Wingate recovery of HRV, HRR1, and HRR2 were similar between groups. In contrast, HRR5 for the WPS group was significantly greater than for the NSmk group. Conclusions: Long-term WP smoking resulted in an altered resting cardiac autonomic state exemplified by reduced overall HRV, specifically, a decline in vagal activity and/or relative increase in sympathetic activity that may persist during anaerobic exercise but not during early recovery.

Keywords: heart rate variability, Wingate anaerobic test, heart rate recovery, hookah

\section{Introduction}

Globally, tobacco use accounts for approximately 5 million deaths per year, mainly due to the use of cigarettes (Islami, Torre, \& Jemal, 2015). The causal link between cigarette smoking and early death and disease has long been known, but the health effects of other tobacco use are poorly understood, including tobacco smoking through a waterpipe. Waterpipe (WP) is also known as shisha, hookah and narghile (Cobb, Sahmarani, Eissenberg, \& Shihadeh, 2012). An important difference between cigarette and WP smoking is the type of tobacco combustion; in a cigarette, tobacco burns at several hundred degrees, while in a WP, tobacco is heated at temperatures $<200{ }^{\circ} \mathrm{C}$ (Layoun et al., 2014). Compared to cigarettes, WP smoking is more efficient in delivering toxins to the smoker with a single WP

\footnotetext{
* Address for correspondence: Mehdi Ahmadian, Department of Physical Education and Sport Sciences, Islamic Azad University of Aliabad Katoul, Daneshjo Street, Aliabad Katoul, Golestan, Iran. E-mail: m_ahmadian98@yahoo.com
}

session exposing the smoker to carbon monoxide (CO) and nicotine levels of 3-9 times and 1.7 times greater than cigarettes, respectively (Eissenberg \& Shihadeh, 2009). Furthermore, like cigarette smoking, WP has been linked to reduced birth weight, genetic damage, and respiratory and cardiovascular disease (Cobb et al., 2012), possibly via enhanced exposure to $\mathrm{CO}$ and volatile aldehydes (Shihadeh \& Saleh, 2005).

Despite the linkages between WP smoking and poor health, there are still some common misconceptions with nicotine content during WP smoking presumed to be lower than that of cigarettes and water used in WP smoking working as a filter and removing all hazardous chemicals such as CO, nicotine and tar (Aslam, Saleem, German, \& Qureshi, 2014). These assumptions have led many to believe that WP smoking is not a hazard to their own and others health (Aslam et al., 2014). To date, most studies have examined WP smoking with regards to its toxic content and exposure (Schubert et al., 2011). Relatively few studies have examined the effects of WP smoking on human physiological function including the autonomic nervous and cardiovascular systems. 
Further, to our knowledge, none have examined the impact of WP smoking on physiological function during or following significant physical stress (e.g., high intensity exercise), a condition where impaired cardiac autonomic activity may lead to serious cardiovascular events (Guasch \& Nattel, 2013).

Cardiac autonomic activity can be examined noninvasively via heart rate variability (HRV), a measure of the influences of sympathetic and vagal modulations of the heart (Task Force of the European Society of Cardiology the North American Society of Pacing Electrophysiology, 1996). Depressed HRV has been established as a powerful independent predictor of increased mortality following myocardial infarction and signifies a shift in sympathovagal balance towards sympathetic predominance and decreased vagal activity (Middlekauff, Park, \& Moheimani, 2014). Likewise, heart rate recovery (HRR) after exercise has become a valuable, non-invasive procedure to assess cardiac parasympathetic reactivation with a small HRR indicating poor reactivation and greater risk of future cardiovascular mortality and morbidity (Grad, 2015). Monitoring of HRV and HRR may therefore provide a simple, non-invasive indication of cardiac autonomic activity to assist in identification of normal and abnormal cardiac function and subsequent health status (Currie, Rosen, Millar, McKelvie, \& MacDonald, 2013).

Typically, HRV and HRR have been examined following moderate or maximal exercise (Ahmadian, Roshan, \& Hosseinzadeh, 2015; Goulopoulou, Fernhall, \& Kanaley, 2009; Goulopoulou et al., 2006; Medeiros, del Rosso, Leicht, Hautala, \& Boullosa, 2017; Perkins, Jelinek, Al-Aubaidy, \& de Jong, 2017). However, a few studies have investigated HRV and HRR following high intensity exercise (e.g., Wingate test) with the recovery of the vagal and sympathetic nervous systems dependent upon the exercise intensity undertaken (Pierpont \& Voth, 2004). Previously, HRR and the high frequency power (HF) component of HRV were reported to be slower and less recovered after high intensity exercise compared with low intensity exercise, respectively (Arai et al., 1989). Furthermore, Stuckey et al. (2012) reported that the autonomic balance was shifted to a greater sympathetic and less parasympathetic activation recovery following a Wingate anaerobic test with recovery taking longer than 1 hour (Stuckey et al., 2012). Further, Perkins et al. (2017) suggested that the high intensity interval training have a greater impact on neurocardiac activity than moderate intensity endurance training as indicated by both linear and nonlinear HRV measures.

To the best of our knowledge, no study to date has investigated the influence of long-term WP smoking on cardiac autonomic function at rest and following high intensity anaerobic exercise with the current study aimed at examining these effects. It was hypothesized that long-term WP smoking, like cigarette smoking, would reduce HRV at rest and following high-intensity anaerobic exercise compared to healthy subjects, indicative of a potentially abnormal state and greater risk of future cardiac events for WP smokers.

\section{Methods}

\section{Study design}

Participants attended one visit at the laboratory between 07:00 and 11:00 a.m. after a rested night. Participants were asked to abstain from WP smoking for at least $48 \mathrm{~h}$, from cigarette smoking on the day of the experiment (in order to prevent the acute impact of smoking on measured variables), and from undertaking exercise on the day before the experiment. Participant's mass, height, body mass index (BMI), and body fat percentage (PBF) were assessed using a standardised and validated body composition analysis system (X-Scan Plus II, Jawon Medical, Kyungsan City, Korea). Participants were then familiarized with the testing procedures including correct use of the equipment including the cycle ergometer. Afterwards, participants undertook a rest recording (10 mins) and then performed a 30-s Wingate anaerobic test followed by a 10-min recovery period. Throughout rest, the Wingate anaerobic test and recovery, heart rate (HR) was continuously monitored and recorded by a 12-lead electrocardiography (ECG) at $500 \mathrm{~Hz}$ (Custo cardio 100, Custo med GmbH, Ottobrunn, Germany).

\section{Participants}

After excluding participants who had regular use of cigarettes (i.e., $\geq 5$ cigarettes/day), participants with physician-diagnosed chronic disease (e.g., arthritis, diabetes, hypertension, cancer, heart attack, chronic cough, bronchitis, and abnormal exercise ECG; Koubaa et al., 2015), a total of 20 sedentary males, with no history of regular physical activity, volunteered and participated in this study. The inclusion criteria included: aged $\geq 20$ years and being either a regular WP smoker (defined as current smoking of $\geq 1 \mathrm{WP}$ per day, and an experience of 10 years smoking) or a non-smoker. Participants were categorised into two groups: a WP smoker group (WPS; $n=10$ ), and a non-smoker group (NSmk; $n=10$ ). This study was approved by the Faculty of Physical Education Ethical Research Committee of Islamic Azad University of Aliabad Katoul Branch, Iran, and was performed in accordance with the 1964 Helsinki declaration; informed consent was obtained from all individual participants included in the study. 


\section{Protocol}

Each participant performed the Wingate, 30-s anaerobic test using a standardised protocol (Goulopoulou et al., 2006), a cycle ergometer (Monark model 894e, Monark Exercise, Vansbro, Sweden), and computer software (Monark Anaerobic Test Software version 2.2; Monark Exercise, Vansbro, Sweden). At the beginning of the protocol, the participants cycled at a low cadence with no resistance for 2 mins. The participants were then asked to increase their cadence over the next $10 \mathrm{~s}$ to achieve maximal cadence before a resistance of $7.5 \%$ of their body weight was added to the flywheel. The participants then continued to cycle at their maximal power output and cadence for $30 \mathrm{~s}$. Strong verbal encouragement was given continuously until the termination of the protocol. Participant's peak HR was recorded along with the calculation of peak power (PP), mean power (MP), and fatigue index (FI) using the Monark Anaerobic Test software.

\section{Procedures}

For HRV analysis, the ECG recordings were visually inspected and any non-sinus beats were removed and replaced by the adjacent normal cycle. If three or more ectopic beats were found within any ECG segment, the subject was excluded from analysis (Esco \& Williford, 2013). Only normal to normal RR intervals at rest and at 0-5 mins of recovery were analysed for HRV using a specialised software program (Kubios HRV version 2.0, University of Kuopio, Kuopio, Finland; see Leicht, Crowther, \& Golledge, 2011).

Variables examined included time domain parameters: standard deviation of normal RR intervals (SDNN) which reflects all the cyclic components responsible for variability in the period of recording (Task Force of the European Society of Cardiology the North American Society of Pacing Electrophysiology, 1996) and root mean square of squared successive differences (RMSSD) which reflects parasympathetic activity (Dong, 2016). We also examined nonlinear measures: standard deviation of the instantaneous beat-to-beat RR interval variability (SD1; minor axis of the ellipse) which reflects mainly the parasympathetic input to the heart (Dong, 2016), standard deviation of the continuous long-term RR interval variability (SD2; major axis of the ellipse) which reflects the sympathetic and parasympathetic contributions to the heart (Dong, 2016), and scaling exponents ( $\alpha 1$ and $\alpha 2)$ which represent the correlation properties of RR interval series (Liao et al., 2014).

Participants were allowed to breath spontaneously as prior studies have shown minimal impact of breathing on time-domain and non-linear measures of HRV
(Patwardhan, Evans, Bruce, Eckberg, \& Knapp, 1995; Penttilä et al., 2001).

\section{Heart rate recovery}

Following the Wingate anaerobic test, HR was recorded during recovery with HRR calculated as the difference between peak HR during the Wingate anaerobic test and HR at $1 \mathrm{~min}$ (HRR 1), $2 \mathrm{~min}$ (HRR 2) and 5 mins (HRR 5) following the exercise (Currie et al., 2013).

\section{Statistical analysis}

Data analysis was performed with the SPSS software (Version 20.0 for Windows; SPSS, Chicago, IL, USA). The normality of the entire dataset was confirmed by the Kolmogorov-Smirnov normality test. Group differences for descriptive characteristics were examined via independent $t$-tests. Significant differences between variables were examined using 2-way (time $\times$ group) ANOVA and Tukey's HSD post-hoc tests. Values are presented as mean \pm standard deviation. Statistical significance was set at $\alpha=.05$.

\section{Results}

Both groups were similar in terms of age, height, mass, BMI, and PBF (Table 1). During the Wingate anaerobic test, both groups exhibited similar PP (NSmk, $566 \pm 115 \mathrm{~W}$ vs. WPS, $582 \pm 90 \mathrm{~W}, p=.778)$, MP (WPS, $257 \pm 95 \mathrm{~W}$ vs. NSmk, $195 \pm 59 \mathrm{~W}, p=.178$ ), and FI (WPS, $55.5 \pm 11.3 \%$ vs. NSmk, $65.2 \% \pm 11.8 \%$, $p=.132$ ). However, the WPS group exhibited a higher peak HR during the Wingate anaerobic test compared to the NSmk group $(174.5 \pm 2.5 \mathrm{bpm}$ vs. $164.5 \pm 9.9$ bpm, $p=.017$ ).

At rest, the WPS group exhibited a significantly greater HR compared with the NSmk group with HR increasing during the Wingate anaerobic test and remaining significantly higher during recovery for both groups (Table 2). No interaction effect was

Table 1

Descriptive characteristics (mean $\pm S D$ ) of all participants

\begin{tabular}{lccc}
\hline & $\begin{array}{c}\text { NSmk } \\
(n=10)\end{array}$ & $\begin{array}{c}\text { WPS } \\
(n=10)\end{array}$ & $p$ \\
\hline Age $(\mathrm{yr})$ & $26.1 \pm 3.6$ & $27.6 \pm 3.1$ & .35 \\
Height $(\mathrm{cm})$ & $174.6 \pm 6.7$ & $174.5 \pm 6.4$ & .95 \\
Mass $(\mathrm{kg})$ & $74.1 \pm 14.3$ & $75.9 \pm 10.4$ & .76 \\
BMI $\left(\mathrm{kg} \cdot \mathrm{m}^{-2}\right)$ & $25.2 \pm 3.7$ & $24.7 \pm 2.9$ & .77 \\
PBF $(\%)$ & $18.9 \pm 6.3$ & $21.2 \pm 5.4$ & .44 \\
\hline Note. NSmk = non-smokers; $\quad$ WPS = waterpipe & smokers; \\
BMI = body mass index; PBF = body fat percentage.
\end{tabular}


Table 2

Mean ( $\pm S D$ ) heart rate variability measures prior to (Pre) and following (Post) Wingate test for non-smoker (NSmk) and waterpipe (WPS) groups

\begin{tabular}{|c|c|c|c|c|}
\hline & Rest (Pre) & Recovery (Post) & Time effect & Group effect \\
\hline \multicolumn{5}{|c|}{ HR (bpm) } \\
\hline NSmk & $71.8 \pm 3.7$ & $131.0 \pm 4.4$ & \multirow{2}{*}{ Post $>\mathrm{Pre}^{* * *}$} & \multirow{2}{*}{ WPS > NSmk* } \\
\hline WPS & $82.4 \pm 5.5^{\dagger}$ & $134.7 \pm 5.1$ & & \\
\hline \multicolumn{5}{|c|}{ SDNN (ms) } \\
\hline NSmk & $61.7 \pm 4.7$ & $26.4 \pm 2.9$ & \multirow{2}{*}{ Pre $>$ Post*** } & \\
\hline WPS & $53.3 \pm 5.0^{4+\dagger}$ & $30.1 \pm 4.0$ & & \\
\hline \multicolumn{5}{|c|}{ RMSSD (ms) } \\
\hline NSmk & $29.8 \pm 5.0$ & $6.4 \pm 0.8$ & \multirow{2}{*}{ Pre $>$ Post*** } & \\
\hline WPS & $34.4 \pm 8.1$ & $6.6 \pm 1.5$ & & \\
\hline \multicolumn{5}{|l|}{ SD1 (ms) } \\
\hline NSmk & $20.6 \pm 4.0$ & $6.6 \pm 2.6$ & \multirow{2}{*}{ Pre $>$ Post*** } & \\
\hline WPS & $21.9 \pm 3.9$ & $5.0 \pm 1.1$ & & \\
\hline \multicolumn{5}{|l|}{ SD2 (ms) } \\
\hline NSmk & $72.0 \pm 12.7$ & $29.8 \pm 9.7$ & \multirow{2}{*}{ Pre $>$ Post*** } & \\
\hline WPS & $63.5 \pm 10.4$ & $38.7 \pm 9.5$ & & \\
\hline \multicolumn{5}{|l|}{$\alpha 1$} \\
\hline NSmk & $1.30 \pm 0.2$ & $0.89 \pm 0.42$ & \multirow{2}{*}{ Pre $>$ Post** } & \\
\hline WPS & $1.50 \pm 0.3$ & $0.92 \pm 0.32$ & & \\
\hline \multicolumn{5}{|l|}{$\alpha 2$} \\
\hline NSmk & $0.90 \pm 0.33$ & $1.45 \pm 0.31$ & \multirow{2}{*}{ Post $>$ Pre*** } & \\
\hline WPS & $0.77 \pm 0.30$ & $1.60 \pm 0.30$ & & \\
\hline
\end{tabular}

Note. $\mathrm{HR}=$ heart rate; $\mathrm{SDNN}=$ standard deviation of RR intervals; $\mathrm{RMSSD}=$ square root of the mean squared differences of successive RR intervals; SD1 = standard deviation of instantaneous RR variability; $\mathrm{SD} 2$ = standard deviation of long-term RR variability; $\alpha 1=$ short-term fractal scaling exponent; $\alpha 2=$ longterm fractal scaling exponent. ${ }^{*} p<.05,{ }^{* *} p<.01,{ }^{* * *} p<.001 ;{ }^{\dagger} p<0.05,{ }^{\dagger \dagger} p<.001$ vs. NSmk.

noted for HRV measures except for SDNN which was significantly greater for the NS group at rest (Table 2). A significant main effect for time was noted for HRV measures with all, except $\alpha 2$, reduced during recovery (Table 2). No significant group effects were noted for any HRV measure.

A main effect for time was noted for HRR values with HRR5 values significantly greater than HRR2 values which were significantly greater than HRR1 values (Figure 1). In addition, a main effect for group was identified with HRR values greater for WPS compared to the NSmk group $(55.9 \pm 13.6 \mathrm{bpm}$ vs. $47.8 \pm 12.5 \mathrm{bpm}$, $p=.015)$. An interaction effect was noted with HRR5 for the WPS group significantly greater than the NSmk group (Figure 1). There were no other differences between groups for HRR values.

\section{Discussion}

To the best of our knowledge, this has been the first study to examine cardiac autonomic function (HRV and HRR) at rest and following a Wingate anaerobic test in WP smokers. The main and novel findings of the present investigation were that: 1) the WPS group exhibited greater HR and lower SDNN at rest, and greater peak HR during the Wingate anaerobic test; 2) recovery HRV following the Wingate anaerobic test was similar for the WPS and NS groups; 3 ) the WPS group experienced a significantly greater HRR5 compared to the NSmk group, most likely as a result of the greater exercise HR. Long-term WP smoking altered resting and peak, anaerobic exercise HR with minimal impact on HRV and HRR. The long-term implications of WP smoking on cardiac autonomic function and cardiac risk remain to be clarified.

\section{HR and HRV at rest and during Wingate test}

The greater HR at rest for the WPS group was in line with previous studies where both acute or long-term cigarette and WP smoking have been associated with increased resting HR (Layoun et al., 2014). Cigarette smoking was reported to act on peripheral sympathetic sites, increase circulating levels of catecholamines, 


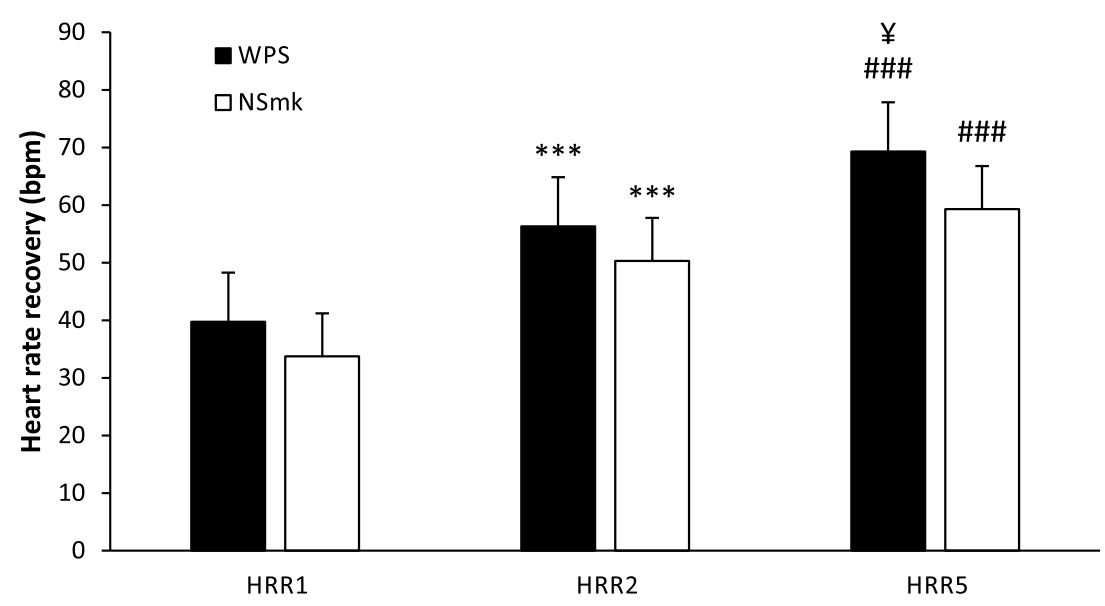

Figure 1. Heart rate recovery after Wingate test for the WPS and NSmk groups. WPS $=$ waterpipe smokers; $\mathrm{NSmk}=$ non-smokers; HRR $1=$ heart rate recovery at $1 \mathrm{~min}$ after exercise; HRR2 = heart rate recovery at 2 mins after exercise; HRR5 = heart rate recovery at 5 mins after exercise.

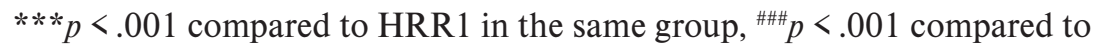
HRR2 in the same group, ${ }^{*} p<.01$ compared to NSmk for the same variable (i.e., HRR5).

augment sympathetic outflow, and result in a long-term reduction of vagal drive (Papathanasiou et al., 2013). This sympathetic predominance was also associated with an impaired baroreflex function, leading to a marked increase in resting HR for smokers compared with non-smokers (Papathanasiou et al., 2013). In the current study, long-term WP smokers exhibited a higher resting HR with reduced SDNN, an overall indicator of HRV and parasympathetic modulations (Task Force of the European Society of Cardiology the North American Society of Pacing Electrophysiology, 1996) compared to non-smokers. Others have reported a similar reduced SDNN at rest for cigarette smokers compared to non-smokers (Barutcu et al., 2005; Levin, Levin, \& Nagoshi, 1992). Collectively, our and prior results indicate a negative impact of cigarette and WP smoking on resting cardiac function (HR) and control (HRV). Given the relationship between reduced HRV and increased cardiac morbidity/mortality (Middlekauff et al., 2014), our results provide further evidence of the negative impact of WP smoking on cardiac health with future studies encouraged to monitor the long-term impact of WP on future cardiac morbidity/mortality.

Interestingly, HR remained elevated for the WPS group during the Wingate anaerobic test. During anaerobic exercise, the increased metabolic demands were met by an increased cardiac output, achieved through an augmentation in HR and stroke volume (Brubaker \& Kitzman, 2011). This HR elevation though was greater for the WPS group likely as a result of the higher initial resting HR and sympathetic modulations for the WPS group. In addition, Walker et al. (1999) reported that plasma epinephrine and norepinephrine levels were increased with smoking that may lead to a significantly greater increase in HR during light physical activity compared to non-smoking. Thus, the higher HR for the WPS group could have been due in part to smoking-induced increases in circulating plasma catecholamines. Irrespective of the mechanisms, the current results have clearly shown that long-term WP smoking led to greater cardiac stress during anaerobic exercise that may increase the risk of cardiac events (Lavie, Milani, Marks, \& de Gruiter, 2001).

\section{Post exercise HR and HRV}

Following the Wingate anaerobic test, HR was elevated with most HRV measures significantly lower compared to resting values for both the WPS and NSmk groups. These findings confirmed previous studies which reported a reduction in HRV following high intensity exercise (Al Haddad, Laursen, Ahmaidi, \& Buchheit, 2009; Buchheit, Laursen, \& Ahmaidi, 2007; Goulopoulou et al., 2009, 2006). For example, Al Haddad et al. (2009) reported that vagal-related HRV indices, immediately post-supramaximal exercise, were significantly lower than immediate pre-supramaximal exercise values. Others reported that HRV was impaired during the first 5, 9, and 10 min after a maximal graded test, a 30-s all-out Wingate test, and following repeated sprint running, respectively (Arai et al., 1989; Buchheit et al., 2007; Goulopoulou et al., 2006). Despite this significant alteration in cardiac autonomic control following 
anaerobic exercise, post-exercise HR and HRV values were similar for the WPS and NSmk groups in the present study. In contrast, previous studies have reported that smoking history was inversely associated with autonomic recovery following graded maximal (Cha, Seo, Ryu, Nam, \& Sung, 2015) and sub-maximal exercise (Cole, Foody, Blackstone, \& Lauer, 2000). It was reported that cardiac autonomic recovery (as measured by HRR3 and HRR5) following maximal aerobic, treadmill exercise was significantly slower in smokers than for non-smokers. This reduced parasympathetic reactivation was suggested to be related to the chronotropic and inotropic effects of catecholamines that were mobilized by nicotine within cigarette smoke (Kobayashi, Takeuchi, Hosoi, \& Loeppky, 2004). Important differences in the research design of the studies including exercise intensity at termination (supramaximal vs. maximal or sub-maximal) may account for the discrepancies between studies. Potentially, a Wingate anaerobic test may be too strenuous and mask the cardiac autonomic differences between WPS and NSmk groups, immediately post-exercise. Longer term, postexercise follow-up following high intensity anaerobic exercise may elucidate on the impact of WPS on postexercise recovery. Further, examination of HRV kinetics following maximal or sub-maximal aerobic exercise may be more appropriate to examine the impact of WPS on cardiac autonomic function with future studies encouraged to investigate this further.

\section{HRR}

As expected, HR declined during recovery from the Wingate anaerobic test with the HRR values greater over time (Al Haddad et al., 2010). The decline of HR during recovery has been reported to be a useful marker of cardiac autonomic control and directly associated with reactivation of post-exercise parasympathetic activity (Papathanasiou et al., 2013). Additionally, the fall in post-exercise HR was considered to reflect the clearance of catecholamines, activation of arterial baroreceptors, and reduction of core body temperature (Goulopoulou et al., 2009). In the current study, HRR1 and HRR2 were similar between WPS and NSmk groups indicating similar parasympathetic reactivation, post anaerobic exercise. Previously Papathanasiou et al. (2013) reported similar HRR following a Bruce maximal treadmill test for smoker and non-smoker males. In contrast, HRR at 3 and 5 mins following maximal aerobic, treadmill exercise was significantly lower for smokers than for non-smokers (Kobayashi et al., 2004). These authors postulated that the smaller HRR and hence reduced parasympathetic reactivation for smokers was due to elevated circulating catecholamines that were a resultant of nicotine within cigarette smoke (Kobayashi et al., 2004). In the present study, catecholamine levels were not assessed with long-term WP expected to enhance these given the reported greater nicotine exposure from WP smoking (Eissenberg \& Shihadeh, 2009). Such enhancements should have resulted in a reduced HRR for the WPS group compared to the NSmk group. As indicated before, the use of high-intensity anaerobic exercise may have been too strenuous to unmask postexercise, cardiac autonomic differences between WPS and NSmk groups.

Nevertheless, HRR5 was significantly greater for the WPS group that may suggest a greater parasympathetic reactivation and restoration of cardiac autonomic activity for long-term WP smokers. This result was surprising given the impact of smoking on nicotine and catecholamine levels (Eissenberg \& Shihadeh, 2009; Kobayashi et al., 2004) and resting HRV in the present and other studies (Barutcu et al., 2005; Levin et al., 1992). However, it should be noted that a direct correlation between the maximum HR achieved at peak exercise and the subsequent HRR has been well reported (Myers, Tan, Abella, Aleti, \& Froelicher, 2007). Therefore, the greater HRR5 value for the WPS group may have resulted from the greater peak HR during the Wingate test for these participants, rather than from greater vagal reactivation. Irrespective of this greater HRR5, the group similarities for HRR1, HRR2 and post-exercise HRV indicated that cardiac autonomic recovery, post-Wingate, was negligibly affected by long-term WP smoking. It remains to be seen whether longer term (> 10 years) WP smoking results in greater changes in HRV and future cardiovascular risk.

This study has confirmed the novel impact of WP smoking on HRV and HRR. However, it is important to note some limitations of the study. Participants were asked to refrain from smoking in order to prevent the acute impact of smoking on measured variables and this may have added additional stress, coupled with pre-exercise stress, on participants, and subsequently affected their mental status. Resting mental status of participants was not recorded in the current study and this should be considered in future studies. Further, prolonged tobacco smoking has been reported to negatively affect respiration (i.e., shortness of breath; Liu et al., 2015) which may influence participants' HRV (i.e., slow breathing rates may increase low frequency power of HRV that may falsely represent sympathetic activation; Bernardi et al., 2000) and ability to perform exercise. Respiratory data was not collected in the current study as several studies have shown minimal impact on breathing on resting, non-linear and time domain measures of HRV (Patwardhan et al., 1995; Penttilä et al., 2001). Further, the exercise bout in the current 
study was anaerobic based with alterations in respiration of minimal importance during the bout (Younes \& Kivinen, 1984). Finally, aerobic fitness levels of participants were not determined in the current study and may be an important factor for HRR and HRV during recovery (Seiler, Haugen, \& Kuffel, 2007). Future studies considering these limitations will elaborate upon the current novel results.

\section{Conclusions}

Long-term WP smoking altered resting cardiac autonomic nervous function, as measured by HRV, with similar post-Wingate, cardiac recovery (HRR) to that of non-smokers. These preliminary results indicate that the impact of long-term ( 10-year) WP smoking on cardiac autonomic control is minimal with future studies recommended to examine the potential deleterious effects of WP smoking on cardiac function and risk. Further, studies of the acute responses of cardiac autonomic function to different types and intensities of exercise (e.g., upper-body, sprinting, maximal, submaximal, etc.) may clarify the impact of WP smoking on cardiac control to a greater extent.

\section{Acknowledgments}

The authors would like to thank the volunteers who took part in this study.

\section{Conflict of interest}

There were no conflicts of interest.

\section{References}

Ahmadian, M., Roshan, V. D., \& Hosseinzadeh, M. (2015). Parasympathetic reactivation in children: Influence of two various modes of exercise. Clinical Autonomic Research, 25, 207-212.

Al Haddad, H., Laursen, P. B., Ahmaidi, S., \& Buchheit, M. (2009). Nocturnal heart rate variability following supramaximal intermittent exercise. International Journal of Sports Physiology and Performance, 4, 435-447.

Al Haddad, H., Laursen, P. B., Chollet, D., Lemaitre, F., Ahmaidi, S., \& Buchheit, M. (2010). Effect of cold or thermoneutral water immersion on post-exercise heart rate recovery and heart rate variability indices. Autonomic Neuroscience: Basic and Clinical, 156, 111-116.

Arai, Y., Saul, J. P., Albrecht, P., Hartley, L. H., Lilly, L. S., Cohen, R. J., \& Colucci, W. S. (1989). Modulation of cardiac autonomic activity during and immediately after exercise. American Journal of Physiology - Heart and Circulatory Physiology, 256, H132-H141.

Aslam, H. M., Saleem, S., German, S., \& Qureshi, W. A. (2014). Harmful effects of shisha: Literature review. International Archives of Medicine, 7, 16.

Barutcu, I., Esen, A. M., Kaya, D., Turkmen, M., Karakaya, O., Melek, M., ... Basaran, Y. (2005). Cigarette smoking and heart rate variability: Dynamic influence of parasympathetic and sympathetic maneuvers. Annals of Noninvasive Electrocardiology, 10, 324-329.

Bernardi, L., Wdowczyk-Szulc, J., Valenti, C., Castoldi, S., Passino, C., Spadacini, G., \& Sleight, P. (2000). Effects of controlled breathing, mental activity and mental stress with or without verbalization on heart rate variability. Journal of the American College of Cardiology, 35, 1462-1469.

Brubaker, P. H., \& Kitzman, D. W. (2011). Chronotropic incompetence: Causes, consequences, and management. Circulation, 123, 1010-1020.

Buchheit, M., Laursen, P. B., \& Ahmaidi, S. (2007). Parasympathetic reactivation after repeated sprint exercise. American Journal of Physiology - Heart and Circulatory Physiology, 293, H133-H141.

Cha, K. S., Seo, M. K., Ryu, H. Y., Nam, J. J., \& Sung, D. J. (2015). Smoking-suppressed heart rate recovery in young male college students who regularly exercised. Iranian Journal of Public Health, 44, 1146-1147.

Cobb, C. O., Sahmarani, K., Eissenberg, T., \& Shihadeh, A. (2012). Acute toxicant exposure and cardiac autonomic dysfunction from smoking a single narghile waterpipe with tobacco and with a "healthy" tobacco-free alternative. Toxicology Letters, 215, 70-75.

Cole, C. R., Foody, J. M., Blackstone, E. H., \& Lauer, M. S. (2000). Heart rate recovery after submaximal exercise testing as a predictor of mortality in a cardiovascularly healthy cohort. Annals of Internal Medicine, 132, 552-555.

Currie, K. D., Rosen, L. M., Millar, P. J., McKelvie, R. S., \& MacDonald, M. J. (2013). Heart rate recovery and heart rate variability are unchanged in patients with coronary artery disease following 12 weeks of high-intensity interval and moderate-intensity endurance exercise training. Applied Physiology, Nutrition, and Metabolism, 38, 644-650.

Dong, J. G. (2016). The role of heart rate variability in sports physiology. Experimental and Therapeutic Medicine, 11, 1531-1536.

Eissenberg, T., \& Shihadeh, A. (2009). Waterpipe tobacco and cigarette smoking: Direct comparison of toxicant exposure. American Journal of Preventive Medicine, 37, 518-523.

Esco, M. R., \& Williford, H. N. (2013). Relationship between post-exercise heart rate variability and skinfold thickness. SpringerPlus, 2, 389.

Goulopoulou, S., Fernhall, B., \& Kanaley, J. A. (2009). Hemodynamic responses and linear and non-linear dynamics of cardiovascular autonomic regulation following supramaximal exercise. European Journal of Applied Physiology, 105, 525-531.

Goulopoulou, S., Heffernan, K. S., Fernhall, B., Yates, G., Baxter-Jones, A., \& Unnithan, V. B. (2006). Heart rate variability during recovery from a Wingate test in adolescent males. Medicine \& Science in Sports \& Exercise, 38, 875-881. 
Grad, C. (2015). Heart rate variability and heart rate recovery as prognostic factors. Clujul Medical, 88, 304-309.

Guasch, E., \& Nattel, S. (2013). CrossTalk proposal: Prolonged intense exercise training does lead to myocardial damage. Journal of Physiology, 591, 4939-4941.

Islami, F., Torre, L. A., \& Jemal, A. (2015). Global trends of lung cancer mortality and smoking prevalence. Translational Lung Cancer Research, 4, 327-338.

Kobayashi, Y., Takeuchi, T., Hosoi, T., \& Loeppky, J. A. (2004). Effects of habitual smoking on cardiorespiratory responses to sub-maximal exercise. Journal of Physiological Anthropology and Applied Human Science, 23, 163-169.

Koubaa, A., Triki, M., Trabelsi, H., Masmoudi, L., Zeghal, K. N., Sahnoun, Z., \& Hakim, A. (2015). Effect of lowintensity continuous training on lung function and cardiorespiratory fitness in both cigarette and hookah smokers. African Health Sciences, 15, 1170-1181.

Lavie, C. J., Milani, R. V., Marks, P., \& de Gruiter, H. (2001). Exercise and the heart: Risks, benefits, and recommendations for providing exercise prescriptions. Ochsner Journal, 3, 207-213.

Layoun, N., Saleh, N., Barbour, B., Awada, S., Rachidi, S., Al-Hajje, A., ... Salameh, P. (2014). Waterpipe effects on pulmonary function and cardiovascular indices: A comparison to cigarette smoking in real life situation. Inhalation Toxicology, 26, 620-627.

Leicht, A. S., Crowther, R. G., \& Golledge, J. (2011). Influence of peripheral arterial disease and supervised walking on heart rate variability. Journal of Vascular Surgery, 54, 1352-1359.

Levin, F. R., Levin, H. R., \& Nagoshi, C. (1992). Autonomic functioning and cigarette smoking: Heart rate spectral analysis. Biological Psychiatry, 31, 639-643.

Liao, F., Brooks, I., Hsieh, C.-W., Rice, I. M., Jankowska, M. M., \& Jan, Y.-K. (2014). Assessing complexity of heart rate variability in people with spinal cord injury using local scale exponents. Conference Proceedings of the IEEE Engineering in Medicine and Biology Society, 2014, 6381-6384.

Liu, Y., Pleasants, R. A., Croft, J. B., Wheaton, A. G., Heidari, K., Malarcher, A. M., ... Strange, C. (2015). Smoking duration, respiratory symptoms, and COPD in adults aged $\geq 45$ years with a smoking history. International Journal of Chronic Obstructive Pulmonary Disease, 10, 1409-1409.

Medeiros, A. R., del Rosso, S., Leicht, A. S., Hautala, A. J., \& Boullosa, D. A. (2017). Methods of assessment of the post-exercise cardiac autonomic recovery: Additional important factors to be considered. International Journal of Cardiology, 239, 23.

Middlekauff, H. R., Park, J., \& Moheimani, R. S. (2014). Adverse effects of cigarette and noncigarette smoke exposure on the autonomic nervous system: Mechanisms and implications for cardiovascular risk. Journal of the American College of Cardiology, 64, 1740-1750.

Myers, J., Tan, S. Y., Abella, J., Aleti, V., \& Froelicher, V. F. (2007). Comparison of the chronotropic response to exercise and heart rate recovery in predicting cardiovascular mortality. European Journal of Cardiovascular Prevention \& Rehabilitation, 14, 215-221.

Papathanasiou, G., Georgakopoulos, D., Papageorgiou, E., Zerva, E., Michalis, L., Kalfakakou, V., \& Evangelou, A. (2013). Effects of smoking on heart rate at rest and during exercise, and on heart rate recovery, in young adults. Hellenic Journal of Cardiolology, 54, 168-177.

Patwardhan, A. R., Evans, J. M., Bruce, E. N., Eckberg, D. L., \& Knapp, C. F. (1995). Voluntary control of breathing does not alter vagal modulation of heart rate. Journal of Applied Physiology, 78, 2087-2094.

Penttilä, J., Helminen, A., Jartti, T., Kuusela, T., Huikuri, H. V., Tulppo, M. P., ... Scheinin, H. (2001). Time domain, geometrical and frequency domain analysis of cardiac vagal outflow: Effects of various respiratory patterns. Clinical Physiology and Functional Imaging, 21, 365-376.

Perkins, S. E., Jelinek, H. F., Al-Aubaidy, H. A., \& de Jong, B. (2017). Immediate and long term effects of endurance and high intensity interval exercise on linear and nonlinear heart rate variability. Journal of Science and Medicine in Sport, 20, 312-316.

Pierpont, G. L., \& Voth, E. J. (2004). Assessing autonomic function by analysis of heart rate recovery from exercise in healthy subjects. American Journal of Cardiology, 94, 64-68.

Seiler, S., Haugen, O., \& Kuffel, E. (2007). Autonomic recovery after exercise in trained athletes: Intensity and duration effects. Medicine \& Science in Sports \& Exercise, 39, 1366-1373.

Schubert, J., Hahn, J., Dettbarn, G., Seidel, A., Luch, A., \& Schulz, T. G. (2011). Mainstream smoke of the waterpipe: Does this environmental matrix reveal as significant source of toxic compounds? Toxicology Letters, 205, 279-284.

Shihadeh, A., \& Saleh, R. (2005). Polycyclic aromatic hydrocarbons, carbon monoxide, "tar", and nicotine in the mainstream smoke aerosol of the narghile water pipe. Food and Chemical Toxicology, 43, 655-661.

Stuckey, M., Tordi, N., Mourot, L., Gurr, L., Rakobowchuk, M., Millar, P., ... Kamath, M. (2012). Autonomic recovery following sprint interval exercise. Scandinavian Journal of Medicine \& Science in Sports, 22, 756-763.

Task Force of the European Society of Cardiology the North American Society of Pacing Electrophysiology. (1996). Heart rate variability: Standards of measurement, physiological interpretation, and clinical use. Circulation, 93, 1043-1065.

Walker, J. F., Collins, L. C., Rowell, P. P., Goldsmith, L. J., Stamford, B. A., \& Moffatt, R. J. (1999). The effect of smoking on energy expenditure and plasma catecholamine and nicotine levels during light physical activity. Nicotine \& Tobacco Research, 1, 365-370.

Younes, M., \& Kivinen, G. (1984). Respiratory mechanics and breathing pattern during and following maximal exercise. Journal of Applied Physiology, 57, 1773-1782. 